\title{
Clozapin und Depotpräparate schützen bei Schizophrenie am besten vor Rückfällen
}

Fragestellung: Wie effektiv sind Antipsychotika unter normalen Behandlungsbedingungen in der Versorgung von schizophrenen Patienten außerhalb von randomisierten kontrollierten Studien?

Hintergrund: Die meisten Daten zur Wirksamkeit von Antipsychotika liegen aus randomisierten kontrollierten Studien vor, mit dem Nachteil, dass sie die normale Behandlungssituation schizophrener Patienten nur ungenügend abbilden, zum Beispiel bezüglich Komorbiditäten, Suizidalität, Chronizität des Verlaufs und Vorbehandlungen. Es ist daher sinnvoll, „Real World“-Studien zur vergleichenden Wirksamkeit von Antipsychotika durchzuführen und die Effektivität der Substanzen zu bestimmen.

Tiihonen J, Mittendorfer-Rutz E, Majak M et al. Real-world effectiveness of antipsychotic treatments in a nationwide cohort of 29.823 patients with schizophrenia. JAMA Psychiatry 2017; 74: 686-93
Patienten und Methodik: Die Autoren brachten zwei Datenbasen zusammen: Eine, in der alle schwedischen Patienten mit einer Schizophreniediagnose und einem Alter zwischen 16 und 64 Jahren im Jahr 2006 ein- geschlossen wurden (29.823 Patienten, davon 4.603 mit kürzlich neu diagnostizierter Erkrankung), und eine zweite Datenbasis, die das Risiko der Rehospitalisierung und des Behandlungsversagens vom 1. Juli 2006 bis 31. Dezember 2013 erfasste. Die Autoren kalkulierten daraus den Hauptoutcome, nämlich das Risiko der Rehospitalisierung und des Behandlungsversagens (definiert als psychiatrische Rehospitalisierung, Suizidversuch, Absetzen oder Umstellen der Medikation oder Tod).

Ergebnisse: Im Beobachtungszeitraum wurden 43,7\% der Patienten rehospitalisiert und bei $71,7 \%$ versagte die Behandlung. Das niedrigste Rehospitalisierungsrisiko wurde unter den Depotpräparaten Haloperidol, Zuclopenthixol, Perphenazin, Olanzapin sowie Clozapin im Vergleich zu keiner Behandlung mit einem Antipsychotikum beobachtet. Eine orale Behandlung mit Flupenthixol, Quetiapin oder Perphenazin war mit dem höchsten Risiko einer Rehospitalisierung verbunden.

Schlussfolgerungen: Clozapin und Depotpräparate von Erstoder Zweitgenerationsantipsychotika sind mit dem niedrigsten Risiko eines Rückfalls verbunden. Die Rehospitalisierungsraten sind bei Depotpräparaten um zirka 20 - $30 \%$ niedriger als unter der Verwendung von oralen Substanzen.

\section{- Kommentar von Klaus Lieb, Mainz}

\section{Quetiapin schneidet wieder schlecht ab}

Die Studie an „Real-world“-Patientendaten hat natürlich viele Schwächen, die in der Natur der Sache liegen, wenn man zwei derartige Datenbasen zusammenbringt. Viele Details gehen verloren. Auf der anderen Seite überzeugt die große Patientenzahl und die Ergebnisse bestätigen die klinische Erfahrung, dass Depotpräparate und Clozapin am besten vor Rückfällen

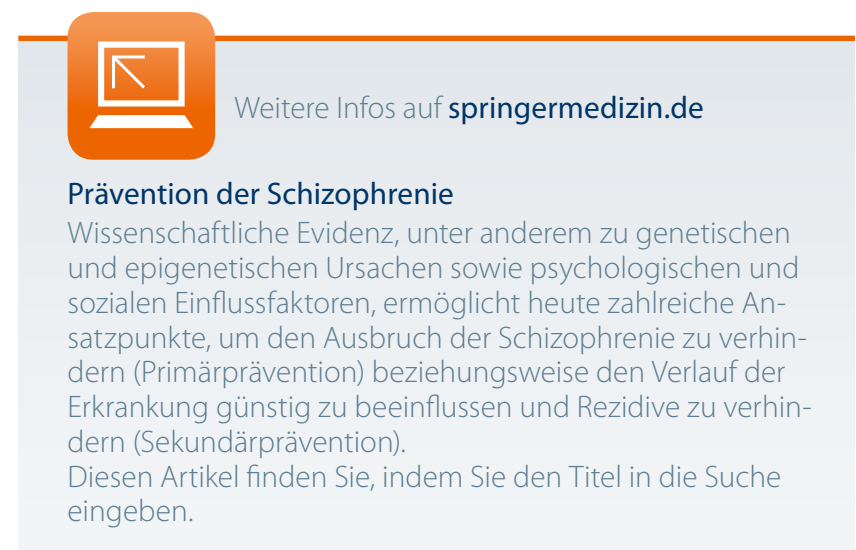

schützen. Dass Quetiapin mit einem relativ hohen Risiko einer Rehospitalisierung assoziiert war, ist nachvollziehbar, da auch die Effizienz in randomisierten kontrollierten Studien mit einer Effektstärke von 0,33-0,44 gegenüber Substanzen wie Risperidon (Effektstärke 0,56) und Amisulprid (Effektstärke 0,66) niedriger ist [1, 2]. In der Versorgungssituation scheint diese Botschaft leider noch nicht angekommen zu sein.
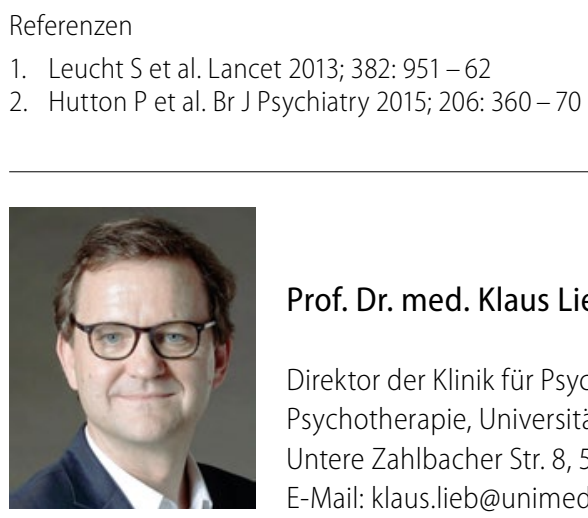

Prof. Dr. med. Klaus Lieb, Mainz

Direktor der Klinik für Psychiatrie und Psychotherapie, Universitätsmedizin Mainz Untere Zahlbacher Str. 8, 55131 Mainz E-Mail: klaus.lieb@unimedizin-mainz.de 